\title{
Systematic review and meta-analysis of
} immunohistochemical prognostic biomarkers in resected oesophageal adenocarcinoma

\author{
L H McCormick Matthews ${ }^{1,6}$, F Noble ${ }^{*, 1,2,6}$, J Tod ${ }^{1}$, E Jaynes ${ }^{3}$, S Harris ${ }^{4}$, J N Primrose ${ }^{1,2}$, C Ottensmeier ${ }^{1,5}$,
} G J Thomas ${ }^{1,3}$ and T J Underwood ${ }^{1,2}$

${ }^{1}$ Cancer Sciences Unit, Faculty of Medicine, University of Southampton, Somers Cancer Research Building, MP824, Southampton SO16 6YD, UK; ${ }^{2}$ Department of Surgery, University Hospital Southampton NHS Foundation Trust, Southampton SO16 6YD, UK; ${ }^{3}$ Department of Cellular Pathology, University Hospital Southampton NHS Foundation Trust, Southampton SO16 6YD, UK; ${ }^{4}$ Public Health Sciences and Medical Statistics, Faculty of Medicine, University of Southampton, Southampton SO16 6YD, UK and ${ }^{5}$ National Institute for Health Research, Experimental Cancer Medicine Centre, Southampton SO16 6YD, UK

Background: Oesophageal adenocarcinoma (OAC) is one of the fastest rising malignancies with continued poor prognosis. Many studies have proposed novel biomarkers but, to date, no immunohistochemical markers of survival after oesophageal resection have entered clinical practice. Here, we systematically review and meta-analyse the published literature, to identify potential biomarkers.

Methods: Relevant articles were identified via Ovid medline 1946-2013. For inclusion, studies had to conform to REporting recommendations for tumor MARKer (REMARK) prognostic study criteria. The primary end-point was a pooled hazard ratio (HR) and variance, summarising the effect of marker expression on prognosis.

Results: A total of 3059 articles were identified. After exclusion of irrelevant titles and abstracts, 214 articles were reviewed in full. Nine molecules had been examined in more than one study (CD3, CD8, COX-2, EGFR, HER2, Ki67, LgR5, p53 and VEGF) and were meta-analysed. Markers with largest survival effects were COX-2 ( $H R=2.47$, confidence interval $(C l)=1.15-3.79), C D 3(H R=0.51$, $95 \% \mathrm{Cl}=0.32-0.70), \mathrm{CD} 8(\mathrm{HR}=0.55, \mathrm{Cl}=0.31-0.80)$ and $\mathrm{EGFR}(\mathrm{HR}=1.65,95 \% \mathrm{Cl}=1.14-2.16)$.

Discussion: Current methods have not delivered clinically useful molecular prognostic biomarkers in OAC. We have highlighted the paucity of good-quality robust studies in this field. A genome-to-protein approach would be better suited for the development and subsequent validation of biomarkers. Large collaborative projects with standardised methodology will be required to generate clinically useful biomarkers.

Oesophageal adenocarcinoma (OAC) is one of the fastest rising cancers in men in the UK and now accounts for more than 5700 new cases per year (Rouvelas et al, 2005; National OesophagoGastric Cancer audit, 2013; Peng et al, 2013). There is an urgent need to identify prognostic subtypes of OAC as despite potentially curative treatment, 5 -year survival is only $35-40 \%$ and current pathological prognostic markers are unreliable. The systematic identification of molecular prognostic markers would allow for improved prognostic information for the patient and a better understanding of the underlying tumour biology. This will help in the logical development of novel targeted therapies for these patients.

*Correspondence: F Noble; E-mail: f.noble@soton.ac.uk

Presented in part (oral) at the International Society for Diseases of the Esophagus annual scientific meeting, Vancouver 2014.

${ }^{6}$ These authors contributed equally to this work.

Received 19 November 2014; Received 22 February 2015; accepted 29 April 2015;

published online 25 June 2015

(c) 2015 Cancer Research UK. All rights reserved 0007-0920/15

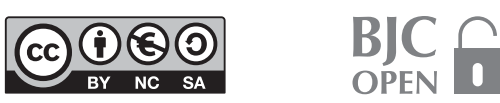


OAC is an area of unmet research need and has been highlighted as a research priority by governments and in the strategies of large funding bodies (Chen et al, 2012). Predicting prognosis after potentially curative surgery for OAC is difficult and inaccurate. It is currently based on internationally accepted tumour staging (Tumour Node Metastasis), with the addition of other important pathological criteria including resection margin status, the presence of vascular or neural invasion and of signet ring histology (Sobin et al, 2010; O'Neill et al, 2013; Schoppmann et al, 2013b; Yendamuri et al, 2013). It is now well recognised that in addition to pathological scoring systems other features of tumours, for example, constituents of the microenvironment, immune infiltration and response to neoadjuvant chemotherapy (NAC), are critical to tumour progression (Courrech Staal et al, 2011). A good response to NAC has been consistently shown to predict for better outcome (Mandard et al, 1994; Fareed et al, 2009; Noble et al, 2013). This is being used by some clinicians to determine adjuvant treatment protocols, but this can only be accurately determined after oesophageal resection, and even in those patients where a poor local tumour response to NAC is observed, a proportion may benefit from systemic treatment by virtue of nodal downstaging (Noble et al, 2013).

Recent advances in OAC have focused on early diagnosis and understanding the genetic landscape of the disease (Kadri et al, 2010; Liu et al, 2014). Next-generation sequencing studies may ultimately lead to molecular phenotype therapeutics in OAC but the widespread application of near-patient whole-genome sequencing at the time of diagnosis is likely to be many years away (Dulak et al, 2013; Weaver et al, 2014). Immunohistochemical (IHC) analysis of differentially expressed proteins is currently superior to DNA-based biomarkers in terms of availability, labour requirements, determining cellular localisation of a marker and takes into consideration post-transcriptional processing. IHC is routinely used in pathology laboratories to differentiate between subtypes of oesophageal cancer and guides targeted therapy with biological agents in a range of cancer types (DiMaio et al, 2012; Ward et al, 2013). A number of IHC-based prognostic biomarkers have been reported in OAC, but none have entered clinical practice (Waterman et al, 2004; Ong et al, 2013).

In this systematic review and meta-analysis, we sought to carefully assess the available published literature on prognostic IHC biomarkers of survival in the resected tumour from patients with OAC. The objective of the study was to identify prognostic markers to provide improved risk stratification in addition to highlighting molecular targets that could offer strategies for the development of novel therapies for patients with OAC.

\section{MATERIALS AND METHODS}

Identification of literature. The aim of the search was to identify all primary literature examining IHC markers of prognosis in OAC. A search strategy combining Plain Text and Medical Subject Heading terms was developed:

(1) $\left(\right.$ esoph $^{\star}$ OR oeoph ${ }^{*}$ AND (carcinoma OR adenocarcinoma OR cance ${ }^{\star}$ OR neoplas ${ }^{\star}$ OR tumo ${ }^{\star}$ ) AND (Prognos* OR Surviv ${ }^{\star}$ OR Mortal ${ }^{\star}$ ) AND (protei* OR marke* OR biomark ${ }^{\star}$ )

OR

(2) Oesophageal Neoplasms/AND (prognosis/or disease-free survival (DFS)/OR Survival/OR mortality/or 'cause of death'/or fatal outcome/or survival rate/) AND (biological markers/or exp antigens, differentiation/or genetic markers/or exp tumour markers, biological/OR genes/or exp genes, neoplasm/) AND Adenocarcinoma/.

The search term was entered into Ovid MEDLINE (1946 to November 2013) without limits and 3059 articles were returned
(Figure 1). Existing systematic reviews and reference lists were crosschecked for studies missed by the search term. In cases where studies were derived from the same data set, the more recent or most complete article was retained. Only published results are included in this review. The Preferred Reporting Items for Systematic Review and Meta-Analysis were utilised (Moher et al, 2009).

Screening. Two independent reviewers (FN and LMM) examined 3059 study titles. From these study titles, 695 abstracts were brought forward as relevant to this study. On review of the abstracts, potentially eligible full-text articles were retrieved with relevant appendices and Supplementary Information.

Eligibility and data extraction. Full-text articles were reviewed against quality criteria (Table 1) derived from the REporting recommendations for tumour MARKer prognostic studies criteria (REMARK - published guidelines for quality reporting in IHCbased tumour biomarker studies; McShane et al, 2005).

For relevant articles, variables were extracted. These included the following: first name author, IHC target, year of publication, number of cases, primary antibody used, dilution of primary antibody, reference group for statistical analysis, number of positive stained cases, univariate or multivariate analysis, hazard ratio (HR), 95\% confidence interval (CI), $P$-value, location of stain and type of survival (overall survival (OS), cancer-specific survival (CSS) or DFS. Only CSS and OS were pooled in the meta-analysis).

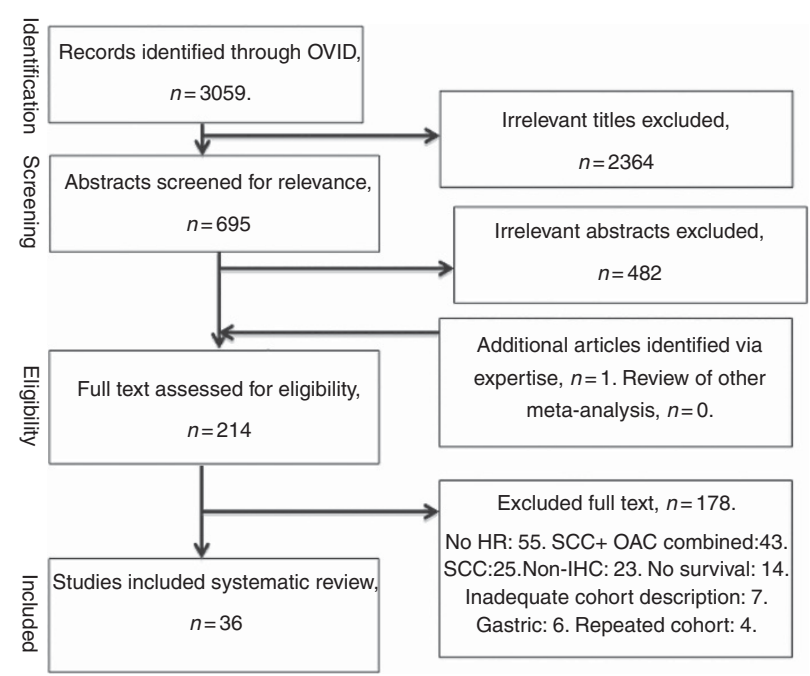

Figure 1. PRISMA flow chart illustrating stages of selection of final articles for meta-analysis.

Table 1. Inclusion criteria adapted from REMARK criteria, utilised at eligibility stage of selection

\begin{tabular}{|c|c|}
\hline 1 & $\begin{array}{l}\text { Prospective or retrospective cohort design with a well-defined study } \\
\text { population with justification for excluded cases }\end{array}$ \\
\hline 2 & Assay of primary/neoadjuvant resected $O A C$ tumour specimens \\
\hline 3 & $\begin{array}{l}\text { Clear description of methods for tissue handling and IHC, including } \\
\text { antigen retrieval, selection, and preparation of both primary and } \\
\text { secondary antibodies, as well as visualisation techniques }\end{array}$ \\
\hline 4 & $\begin{array}{l}\text { A clear statement on the choice of positive and negative controls and } \\
\text { on the outcome of the assay to ensure that the primary antibody used } \\
\text { was a well-validated reagent }\end{array}$ \\
\hline 5 & Statistical analysis using univariate or multivariate hazards modelling \\
\hline 6 & Reporting of the resulting HRs including $95 \% \mathrm{Cls}$ and $P$-values \\
\hline \multicolumn{2}{|r|}{$\begin{array}{l}\text { Abbreviations: } \mathrm{Cl}=\text { confidence interval; } \mathrm{IHC}=\text { immunohistochemical; } \mathrm{HR}=\text { hazard ratio } \\
\mathrm{OAC}=\text { oesophageal adenocarcinoma; } \mathrm{REMARK}=\mathrm{REp} \text { orting recommendations for tumor } \\
\text { MARKer. }\end{array}$} \\
\hline
\end{tabular}




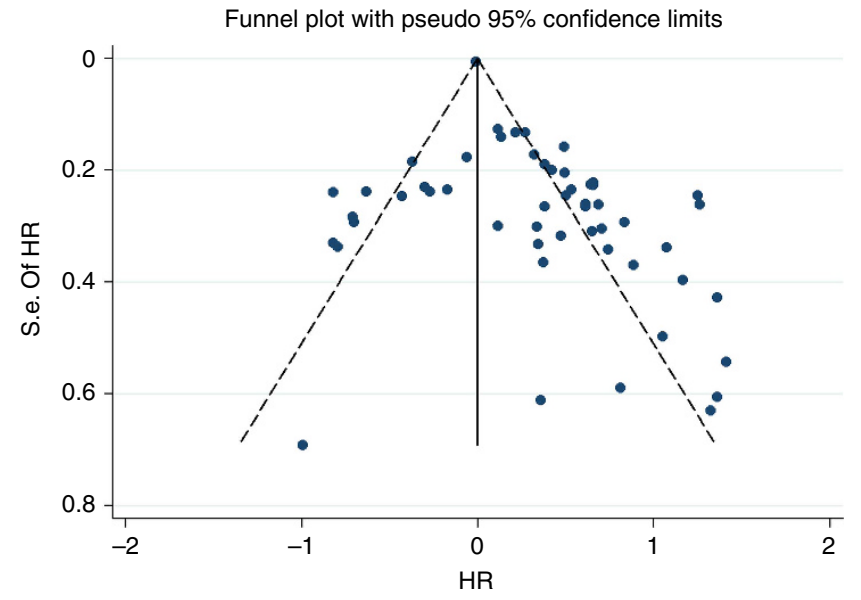

Figure 2. Funnel plot showing publication bias for the 58 included studies providing $\mathrm{HR}$ and $\mathrm{Cl}$. Plotted points are frequently seen away from the ' 0 '.

Synthesis and statistical analysis. Both univariate and multivariate results were considered for the meta-analysis, with univariate analysis used preferentially when both were available. Univariate analysis was preferred due to the variability of analysis used (univariate $n=3$; multivariate $n=5$; and both $n=27$ ). In addition, there was variability in the method and variables used to derive the final multivariate model making comparative analysis across studies biased (Supplementary Figure 1). Of 36 studies included in the review, 27 (75\%) stated HR and CI derived by multivariate analysis. Of these, the method used to make the model was described in only nine $(33 \%)$. The method used to make the model varied as follows: entering all variables on univariate analysis into the model in 6 (22\%); using backward stepwise regression in $2(7 \%)$ and it was impossible to accurately assess the method used in 19 (70\%). The number of variables used to create the multivariate model varied and was anywhere between 3 and 13 . Where studies considered opposite degrees of expression, the inverse $\mathrm{HR}$ and $\mathrm{CI}$ was calculated to give results for high expression. For biomarkers analysed in more than one study, HR and CIs were entered into a random-effects model on Stata Statistical Software, SE 12 (StataCorp LP, College Station, TX, USA). The synthesised HR is reported as increase of risk of death from OAC within the individual study's reference group with $\mathrm{HR}>1$ indicating increased risk of death, and $\mathrm{HR}<1$ indicating decreased risk of death.

The heterogeneity of results between studies was assessed using I2 statistics (a measure of consistency of results between studies) with increasing heterogeneity implying less utility in generalising across studies (Higgins et al, 2003). Sensitivity analysis was carried out by removing individual studies from the meta-analysis and assessing the effect on the pooled result. Presence of publication bias was formally evaluated using funnel plots (Figure 2) (Sterne et al, 2001).

\section{RESULTS}

Excluded studies. Of the 3059 articles returned, 2364 were excluded on review of title and 482 on examination of abstract, leaving 214 articles considered relevant. Crosschecking of existing systematic review reference lists revealed no further relevant articles (Vallbohmer and Lenz, 2006; Ong et al, 2010; Chan et al, 2012; Chen et al, 2012, 2013; Peng et al, 2013; Gowryshankar et al, 2014).
Upon careful review of the 214 articles against the REMARK inclusion criteria, 56 did not provide a HR, 43 combined OAC and SCC subtypes for statistical analysis, 25 examined only SCC, 23 used non-IHC methodology, 14 did not examine survival, 7 had inadequate cohort description, 6 examined gastric cancer and 4 repeated use of a cohort. This left only 36 articles that conformed to REMARK inclusion criteria.

Included studies. 26 individual research centres contributed to the 36 articles. 20 studies (56\%) reported cohorts of patients who underwent surgery only; in 6 studies (17\%) the authors reported that some patients had undergone neoadjuvant therapy and in 10 studies (28\%) no information were given regarding preoperative treatment. Of the six studies, where some kind of neoadjuvant treatment was reported, this consisted of chemoradiotherapy in five (14\%) and chemotherapy in three (8\%). The percentage of patients who had undergone neoadjuvant therapy varied from 8 to $100 \%$. The specific neoadjuvant treatment regimes that were used were only reported in three out of six (50\%) studies. Little overlap in methodology was seen with every centre using different antibodies at different dilutions or different scoring systems. Variable cohort sizes were used, ranging from 24 to 259 cases. 50 HRs, CIs and relevant variables were extracted from these studies. Extracted data is reported in Table 2 with biomarkers grouped according to the hallmark of cancer with which a functional role for that molecule has been most closely attributed (Figure 3; Hanahan and Weinberg, 2011).

19 of the 36 articles examined one or more of the same nine molecules, making them suitable for meta-analysis. Upon pooling studies, six of the nine molecules showed prognostic significance: COX-2, CD3, CD8, p53, EGFR and HER2 in order of HR, with LgR5, VEGF and Ki67 not reaching significance. Forrest plots are shown in Figure 4.

In three of the studies included in the meta-analyses, only multivariate analysis was stated. Where sensitivity analysis was not possible due to lack of appropriate, robust literature, agreement on prognostic value was considered with other studies on OAC.

COX-2. COX-2 is a rate-limiting enzyme in the conversion of arachidonic acid to prostaglandins and has multiple functions in immune evasion, angiogenesis and proliferation. COX-2 is consistently detected with varying expression in OAC (Lagorce et al, 2003). Subsequently, inhibitors of COX-2 have been shown to be protective of progression from Barrett's oesophagus to OAC, and have shown some promise in improving prognosis when used alongside NAC (Corley et al, 2003; Tuynman et al, 2005).

Three studies, consisting of a total of 382 patients, contributed to quantify the effect of COX-2, which was found to correlate negatively with prognosis (Buskens et al, 2002; Bhandari et al, 2006; Prins et al, 2012). Although consistent overexpression is noted in OAC, differences in cutoff values for staining positivity, and variability in numbers of positive staining cases between studies are seen here (27\% and $79 \%$ positive) (Buskens et al, 2002; Prins et al, 2012). Within other prognostic studies on OAC, not providing HR, both significant (three studies, $n=194$ ) and nonsignificant (three studies, $n=139$ ) results have been reported (Lagorce et al, 2003; France et al, 2004; Kulke et al, 2004; Heeren et al, 2005; Mobius et al, 2005; Tuynman et al, 2008).

CD3. CD3 + cells are mature T lymphocytes and quantification of $\mathrm{CD} 3+$ has been commonly used to evaluate immunological response against solid tumours (Dahlin et al, 2011). Two studies identified $\mathrm{CD} 3$ as an independent predictor of improved survival in OAC (Rauser et al, 2010; Zingg et al, 2010). Methods of exploration varied; with one study using an automated scoring system across 10 random high-power fields $v s$ central CD3 + lymphocyte count (Rauser et al, 2010; Zingg et al, 2010). However, the studies show good agreement $\left(I^{2}=0.00 \%\right)$ and similar weighting on meta-analysis. 
Table 2. Extracted data from biomarker articles

\begin{tabular}{|c|c|c|c|c|c|c|c|c|c|c|}
\hline & $\begin{array}{c}\mathrm{IHC} \\
\text { target }\end{array}$ & $N$ & $\begin{array}{l}\text { Positive } \\
\text { cases }\end{array}$ & $\begin{array}{l}\text { Primary } \\
\text { antibody } \\
\text { (dilution) }\end{array}$ & $\begin{array}{c}\text { Reference } \\
\text { group }\end{array}$ & $\begin{array}{c}\text { Uni or } \\
\text { Multivariate }\end{array}$ & Survival & HR & $\mathrm{Cl}$ & $P$-value \\
\hline \multicolumn{11}{|c|}{ Evasion of growth supressors } \\
\hline Evangelou et al, 2008 & Caspase-3 & 35 & NA & $\begin{array}{l}\text { Cell signalling } \\
\text { Bioline }(1: 100)\end{array}$ & Labelling index & Univariate & DFS & $<0.01^{\mathrm{a}}$ & $0.298-3.302^{\mathrm{a}}$ & 0.990 \\
\hline Evangelou et al, 2008 & E2f-1 & 35 & $23(66 \%)$ & Santa Cruz $(1: 100)$ & $>35 \%$ cells labelling index & Univariate & DFS & 3.908 & $0.153-0.992^{\mathrm{a}}$ & 0.048 \\
\hline Cavazzola et al, 2009 & p53 & 38 & $24(52 \%)$ & $\begin{array}{l}\text { Sigma Biosciences } \\
(1: 100)\end{array}$ & $>10 \%$ & Multivariate & CSS & 1.429 & $0.429-4.725$ & 0.514 \\
\hline Moskaluk et al, 1996 & p53 & 88 & $40(45 \%)$ & Novocastra (NA) & $>50 \%$ & Univariate & OS & 1.46 & $0.87-2.46$ & 0.155 \\
\hline Madani et al, 2010 & p53 & 142 & $48(34 \%)$ & Dako $(1: 50)$ & +2 to +8 & Univariate & OS & 1.64 & $1.1-2.45$ & 0.014 \\
\hline \multicolumn{11}{|c|}{ Sustained Proliferative signalling } \\
\hline Langer et al, 2006 & EGFR & 137 & $72(53 \%)$ & Cytomed $(1: 60)$ & $>10 \%$ cells + ve. & Univariate & OS & 0.99 & $0.98-1.00^{\mathrm{a}}$ & 0.039 \\
\hline Ong et al, 2013 & EGFR & 359 & $36(10 \%)$ & Novocastra $(1: 10)$ & $+2,+3$ & Univariate & OS & 1.520 & $1.03-2.26$ & 0.040 \\
\hline Ong et al, $2013^{b}$ & EGFR & 663 & $\begin{array}{l}\text { NA } \\
-100\end{array}$ & Novocastra $(1: 10)$ & +1 & Univariate & OS & 0.83 & $0.66-1.04$ & .10 \\
\hline & & & 100 (15\%) & & $\begin{array}{l}+2 \\
+3\end{array}$ & & & $\begin{array}{l}1.41 \\
0.94\end{array}$ & $\begin{array}{r}.05-1.91 \\
0.58-1.52\end{array}$ & $\begin{array}{l}0.02 \\
0.80\end{array}$ \\
\hline Wang et al, 2007 & EGFR & 103 & 33 (32\%) & Dako (NA) & $>5 \%$ & Univariate & os & 1.93 & $1.24-3.02$ & 0.004 \\
\hline Nakamura et al, 1994 & HER2 & 62 & $15(19 \%)$ & $\begin{array}{l}\text { Boehringer } \\
\text { Mannheim } \\
\text { Biochemica (NA) }\end{array}$ & +2 & Univariate & OS & 4.100 & $1.4-11.8$ & 0.015 \\
\hline Yoon et al, 2012 & HER2 & 708 & $119(17 \%)$ & Herceptest (NA) & $+2,+3$ & Univariate & OS & 0.760 & $0.59-0.96$ & 0.024 \\
\hline Phillips et al, 2013 & HER2 & 135 & 31 (23\%) & Ventana (NA) & $+2,+3$ & Multivariate & OS & 0.840 & $0.53-1.33$ & 0.470 \\
\hline Dutta et al, 2012 & Ki67 & 98 & NA & Dako $(1: 50)$ & $\begin{array}{l}\text { Slidepath scoring } \\
\text { algorithm. Tertiles. }\end{array}$ & Univariate & CSS & 1.460 & $1.01-2.12$ & 0.048 \\
\hline Evangelou et al, 2008 & Ki67 & 35 & NA & Dako (1: 100) & $\begin{array}{l}<35 \% \text { cells }+ \text { ve, labelling } \\
\text { index }\end{array}$ & Univariate & DFS & 3.757 & $0.986-11.68$ & 0.050 \\
\hline Falkenback et al, 2008 & Ki67 & 59 & $50(85 \%)$ & Dako $(1: 1000)$ & $0-10 \%$ & Univariate & CSS & 3.900 & $1.7-9.1$ & $<0.001$ \\
\hline Tuynman et al, 2008 & MET & 145 & 78 (54\%) & Zymed (1:100) & $+2,+3$ & Univariate & DFS & 2.300 & $1.3-4.1$ & 0.004 \\
\hline Prins et al, 2013 & $\mathrm{p}$-mTOR & 147 & $29(19.7 \%)$ & $\begin{array}{l}\text { Cell Signalling } \\
\text { Technology }(1: 50)\end{array}$ & $2+, 3+$ & Univariate & CSS & 1.648 & $1.019-2.664$ & 0.042 \\
\hline Ong et al, 2013 & PAPSS2 & 337 & $216(64 \%)$ & Abcam $(1: 600)$ & $+2,+3$ & Univariate & OS & 1.240 & $0.96-1.61$ & 0.100 \\
\hline Schoppmann et al, 2012 & pSTAT3 & 179 & $72(40 \%)$ & $\begin{array}{l}\text { Cell Signalling } \\
\text { Technology } \\
(1: 100)\end{array}$ & $>10$ (>median) & Univariate & OS & 1.982 & $1.186-3.311$ & 0.050 \\
\hline Bettstetter et al, 2013 & PTEN & 117 & $101(86 \%)$ & $\begin{array}{l}\text { Cell Signalling } \\
\text { Technology }(1: 50)\end{array}$ & $>75 \%$ & Multivariate & OS & 0.451 & $0.233-0.873$ & 0.018 \\
\hline \multicolumn{11}{|c|}{ Escape from immune surveillance } \\
\hline Loos et al, 2011 & $\mathrm{~B} 7-\mathrm{H} 1$ & 101 & $74(73 \%)$ & Abcam (NA) & $\begin{array}{c}>+4 \\
\begin{array}{c}\text { (Intensity }+ \text { proportion of } \\
\text { cells) }\end{array}\end{array}$ & Univariate & OS & 2.92 & $1.50-5.66$ & $<0.001$ \\
\hline Rauser et al, 2010 & CD3 & 99 & $57(58 \%)$ & $\begin{array}{l}\text { NeoMarkers } \\
(1: 100)\end{array}$ & >2.0 Labelling indices & Univariate & OS & 0.49 & $0.28-0.85$ & 0.012 \\
\hline Zingg et al, 2010 & CD3 central & 105 & NA & Dako $(1: 50)$ & $>563$ (>median count) & Univariate & OS & 0.53 & $0.33-0.84$ & 0.008 \\
\hline Zingg et al, 2010 & CD4 central & 105 & 58 (55\%) & NeoMarkers $(1: 40)$ & $>30$ (>median count) & Univariate & OS & 0.74 & $0.47-1.16$ & 0.187 \\
\hline Zingg et al, 2010 & CD25 central & 105 & NA & NeoMarkers $(1: 10)$ & $>33$ (>median count) & Univariate & OS & 0.76 & $0.48-1.22$ & 0.262 \\
\hline Zingg et al, 2010 & CD8 central & 105 & $51(49 \%)$ & Dako $(1: 50)$ & $>225$ (>median count) & Univariate & OS & 0.44 & $0.27-0.69$ & $<0.001$ \\
\hline Dutta et al, 2012 & CD8 tertiles & 98 & NA & Dako (1: 100) & Slidepath scoring algorithm & Univariate & CSS & 0.69 & $0.48-0.99$ & 0.048 \\
\hline Rauser et al, 2010 & CD45RO & 110 & 93 (85\%) & Dako (1:1200) & $>0.9$ Labelling indices & Univariate & DFS & 0.44 & $0.23-0.84$ & 0.013 \\
\hline Dutta et al, 2012 & CD68 & 98 & NA & Dako $(1: 200)$ & $\begin{array}{l}\text { Slidepath scoring } \\
\text { algorithm. Tertiles. }\end{array}$ & Univariate & CSS & 1.38 & $0.99-1.94$ & 0.061 \\
\hline Zingg et al, 2010 & FoxP3 central & 105 & $46(43 \%)$ & eBioscience $(1: 50)$ & $>117$ (>median count) & Univariate & OS & 0.65 & $0.40-1.05$ & 0.079 \\
\hline \multicolumn{11}{|c|}{ Deregulation of cellular energetics } \\
\hline Birner et al, 2011 & CAIX & 182 & $85(47 \%)$ & Abcam $(1: 1000)$ & $\begin{array}{l}>\text { median score }(20 \text { out of } \\
\text { score } 0-300)\end{array}$ & Univariate & OS & 1.844 & $1.11-3.08$ & 0.007 \\
\hline \multicolumn{11}{|c|}{ Tumour promoting inflammation } \\
\hline Wang et al, 2006 & ANXA1 & 104 & $41(39 \%)$ & $\begin{array}{l}\text { BD Biosciences } \\
(1: 100)\end{array}$ & $>25 \%$ & Univariate & OS & 1.930 & $1.25-2.99$ & 0.003 \\
\hline Bhandari et al, 2006 & $\operatorname{cox}-2$ & 90 & NA & $\begin{array}{l}\text { Cayman Chemical } \\
(1: 100)\end{array}$ & $>200$ & Univariate & CSS & 3.530 & $2.11-5.89$ & $<0.001$ \\
\hline Buskens et al, 2002 & $\operatorname{cox}-2$ & 145 & $115(79 \%)$ & $\begin{array}{l}\text { Cayman Chemical } \\
(1: 200)\end{array}$ & $+2,+3$ & Univariate & OS & 3.200 & $1.5-7.1$ & 0.002 \\
\hline Prins et al, 2012 & $\operatorname{cox}-2$ & 147 & $39(27 \%)$ & $\begin{array}{l}\text { Cayman Chemical } \\
(1: 100)\end{array}$ & +3 & Univariate & OS & 1.700 & $1.07-2.69$ & 0.023 \\
\hline Tuynman et al, 2008 & $\operatorname{cox}-2$ & 145 & 78 (54\%) & $\begin{array}{l}\text { Cayman Chemical } \\
(1: 200)\end{array}$ & $+2,+3$ & Univariate & DFS & 1.400 & $0.8-2.6$ & 0.234 \\
\hline \multicolumn{11}{|c|}{ Evasion of apoptosis } \\
\hline Ong et al, 2013 & DCK & 355 & $126(36 \%)$ & \begin{tabular}{|l|} 
Lifespan \\
Biosciences $(1: 10)$
\end{tabular} & $+2,+3$ & Univariate & OS & 0.980 & $0.75-1.28$ & 0.860 \\
\hline Chandra et al, 2002 & $\mathrm{GST} \pi$ & 15 & $6(40 \%)$ & Vector laboratories & $3+$ & Univariate & DFS & 2.250 & $0.71-7.17$ & 0.350 \\
\hline Ong et al, 2013 & MTMR9 & 356 & $88(25 \%)$ & Novus $(1: 350)$ & $+2,+3$ & Univariate & OS & 1.140 & $0.87-1.51$ & 0.340 \\
\hline Ong et al, 2013 & NEIL2 & 357 & $198(55 \%)$ & $\begin{array}{l}\text { Sigma-Aldrich } \\
(1: 50)\end{array}$ & $+2,+3$ & Univariate & OS & 1.120 & $0.87-1.43$ & 0.390 \\
\hline Ong et al, 2013 & SIRT2 & 359 & $156(44 \%)$ & $\begin{array}{l}\text { Atlas Antibodies } \\
(1: 100)\end{array}$ & $+2,+3$ & Univariate & OS & 1.310 & $1.03-1.67$ & 0.030 \\
\hline Ong et al, $2013^{b}$ & SIRT2 & 663 & NA & $\begin{array}{l}\text { Atlas Antibodies } \\
(1: 100)\end{array}$ & 2 & Univariate & OS & 1.69 & $1.10-2.60$ & 0.02 \\
\hline & & & $290(44)$ & 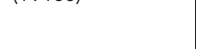 & 1 & & & 1.81 & $1.24-2.64$ & $<0.01$ \\
\hline Ong et al, 2013 & WT1 & 358 & $19(5 \%)$ & Dako $(1: 800)$ & $\begin{array}{c}0 \\
+2,+3\end{array}$ & Univariate & OS & $\begin{array}{l}1.37 \\
0.710\end{array}$ & $\begin{array}{l}0.96-1.97 \\
0.39-1.30\end{array}$ & $\begin{array}{l}0.08 \\
0.270\end{array}$ \\
\hline
\end{tabular}




\section{Table 2. (Continued)}

\begin{tabular}{|c|c|c|c|c|c|c|c|c|c|c|}
\hline & $\begin{array}{c}\mathrm{IHC} \\
\text { target }\end{array}$ & $\mathbf{N}$ & $\begin{array}{c}\text { Positive } \\
\text { cases }\end{array}$ & $\begin{array}{l}\text { Primary } \\
\text { antibody } \\
\text { (dilution) }\end{array}$ & $\begin{array}{l}\text { Reference } \\
\text { group }\end{array}$ & $\begin{array}{c}\text { Uni or } \\
\text { Multivariate }\end{array}$ & Survival & HR & $\mathrm{Cl}$ & $P$-value \\
\hline \multicolumn{11}{|c|}{ Inducing angiogenesis } \\
\hline Dutta et al, 2012 & CD34 & 98 & NA & Dako $(1: 150)$ & $\begin{array}{l}\text { Slidepath scoring } \\
\text { algorithm. Tertiles. }\end{array}$ & Univariate & CSS & 0.94 & $0.67-1.34$ & 0.736 \\
\hline Cavazzola et al, 2009 & VEGF & 38 & 22 (48\%) & Santa Cruz $(1: 400)$ & $>30 \%$ cells stained & Multivariate & CSS & 0.369 & $0.095-1.436$ & 0.115 \\
\hline Prins et al, 2012 & VEGF & 143 & $90(63 \%)$ & $\begin{array}{l}\text { R\&D systems } \\
(1: 50)\end{array}$ & $>+1$ & Univariate & CSS & 1.900 & $1.22-2.96$ & 0.005 \\
\hline Xie et al, 2013 & VEGF-C & 128 & 96 (75\%) & Santa Cruz $(1: 50)$ & $\begin{array}{l}>0.18 \text { Mean optical } \\
\text { density }\end{array}$ & Multivariate & DFS & 3.491 & $2.156-5.652$ & $<0.0001$ \\
\hline \multicolumn{11}{|c|}{ Tissue invasion and metastasis } \\
\hline Hector et al, 2010 & $\overline{A X L}$ & 92 & $56(61 \%)$ & $\begin{array}{l}\text { R\&D systems } \\
(1: 100)\end{array}$ & +3 & Multivariate & OS & 1.91 & $1.04-3.49$ & 0.036 \\
\hline Falkenback et al, 2008 & E-cadherin & 59 & $44(75 \%)$ & Dako $(1: 100)$ & Absent/reduced & Univariate & CSS & 3.900 & $1.2-12.9$ & 0.017 \\
\hline Becker et al, 2010 & LgR5 & 24 & NA & $\begin{array}{l}\text { MBL Internation } \\
\text { Co }(1: 50)\end{array}$ & $>5$ (Intensity + proportion) & Univariate & OS & 2.860 & $1.08-7.61$ & 0.040 \\
\hline von Rahden et al, 2011 & LgR5 & 60 & $51(85 \%)$ & Abcam (NA) & $>15 \%$ & Univariate & OS & 2.418 & $1.17-4.99$ & 0.033 \\
\hline Grimm et al, 2010 & MMP-1 & 60 & 33 (55\%) & $\begin{array}{l}\text { Hiddenhausen } \\
\text { (NA) }\end{array}$ & $>46 \%$ & Univariate & OS & 1.453 & $0.7101-2.9718$ & 0.307 \\
\hline Streppel et al, 2012 & Mucin 16 & 95 & $66(70 \%)$ & Abcam $(1: 200)$ & Moderate/Diffuse & Univariate & NA & 1.410 & $0.734-2.709$ & 0.303 \\
\hline Wijnhoven et al, 2005 & p120 & 96 & $65(67 \%)$ & $\begin{array}{l}\text { Transduction } \\
\text { laboratories } \\
(1: 1000)\end{array}$ & $<90 \%$ & Multiivariate & OS & 2.100 & $1.1-4.2$ & 0.006 \\
\hline $\begin{array}{l}\text { Schoppmann et al, } \\
\text { 2013b }\end{array}$ & $\begin{array}{c}\text { Podoplanin } \\
\text { (lymphovascular } \\
\text { invasion) }\end{array}$ & 194 & 81 (42\%) & Ventana (NA) & $\begin{array}{c}\text { Tumour cluster in } \\
\text { podoplanin decorated } \\
\text { space }\end{array}$ & Univariate & OS & 1.863 & $1.086-0.195^{\mathrm{a}}$ & $<0.01$ \\
\hline $\begin{array}{l}\text { Schoppmann et al, } \\
2013 a\end{array}$ & $\begin{array}{l}\text { Podoplanin } \\
\text { (CAFs) }\end{array}$ & 200 & 118 (59\%) & Venatana $(1: 300)$ & $>10 \%$ CAFs & Univariate & OS & 1.843 & $1.097-3.096$ & 0.001 \\
\hline Birner et al, 2012 & RKIP & 179 & NA & $\begin{array}{l}\text { Upstage/Millipore } \\
(1: 1000)\end{array}$ & $\begin{array}{c}>80 \text { out of score } 0-300 \\
\quad(>\text { median score })\end{array}$ & Multivariate & DFS & 0.494 & $0.278-0.878$ & 0.016 \\
\hline Ong et al, 2013 & TRIMM44 & 349 & $197(56 \%)$ & $\begin{array}{l}\text { Protein Tech group } \\
(1: 50)\end{array}$ & +2 & Univariate & OS & 1.310 & $1.01-1.70$ & 0.040 \\
\hline Ong et al, $2013^{b}$ & TRIMM44 & 655 & & $\begin{array}{l}\text { Protein Tech group } \\
(1: 50)\end{array}$ & & Univariate & OS & 1.46 & $0.89-2.44$ & 0.4 \\
\hline & & & 442 (67\%) & & $\begin{array}{l}+2 \\
+3\end{array}$ & & & $\begin{array}{l}1.59 \\
1.94\end{array}$ & $\begin{array}{l}0.96-2.63 \\
1.09-3.44\end{array}$ & $\begin{array}{l}0.07 \\
0.02\end{array}$ \\
\hline Laerum et al, 2012 & $\begin{array}{l}\text { uPAR (Cancer } \\
\text { cells) }\end{array}$ & 60 & $37(62 \%)$ & Raised in-house & $+2,+3,+4$ & Univariate & OS & 2.020 & $1.11-3.66$ & 0.021 \\
\hline Laerum et al, 2012 & $\begin{array}{c}\text { UPAR } \\
\text { (Macrophages) }\end{array}$ & 60 & $57(95 \%)$ & Raised in-house & $+2,+3,+4$ & Univariate & OS & 1.120 & $0.62-2.01$ & 0.710 \\
\hline Laerum et al, 2012 & $\begin{array}{c}\text { uPAR } \\
\text { (myofibroblasts) }\end{array}$ & 60 & $39(65 \%)$ & Raised in-house & $+2,+3,+4$ & Univariate & OS & 1.600 & $0.86-2.99$ & 0.140 \\
\hline
\end{tabular}

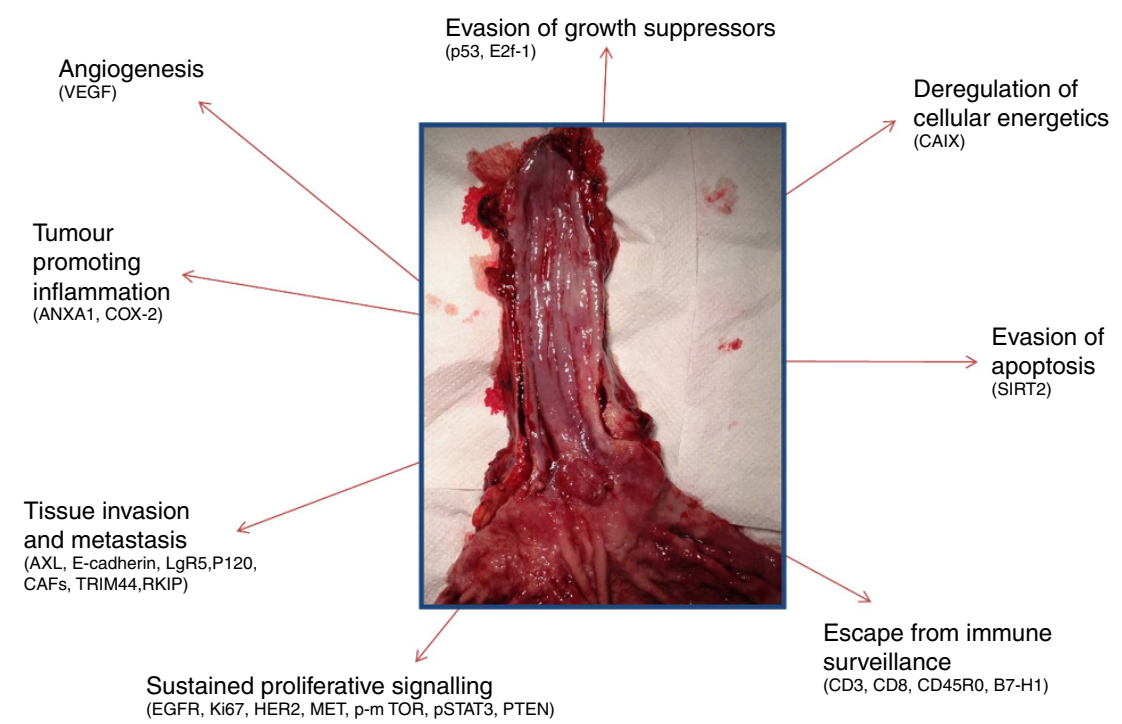

Figure 3. Statistically significant prognostic biomarkers from at least one study in resected oesophageal adenocarcinoma covering all hallmarks of cancer.

CD8. CD8 is a marker of cytotoxic T cells. CD8 + cells kill cancer cells via release of granzyme and perforin or via Fas ligand presentation (Owen et al, 2013). This is an area of considerable interest with trials in a number of solid organ cancers examining strategies to enhance tumour-cell killing. The discovery of the role of PD-L1 on tumour cells and its interaction with the PD-1 receptor on cyctotoxic $\mathrm{T}$ cells leading to immune cell exhaustion have led to the development of antibodies targeting both the 
COX2:

\begin{tabular}{|c|c|c|c|}
\hline Study & Hazard ratio & $95 \%$ confidence interval & Weight (\%) \\
\hline Buskens CJ, 2002 & 3.20 & $1.50-7.10$ & 16.79 \\
\hline Prins MJ, 2012 & 1.70 & $1.07-2.69$ & 54.67 \\
\hline Bhandari P, 2006 & 3.53 & $2.11-5.89$ & 28.54 \\
\hline \multicolumn{2}{|c|}{ Overall: HR 2.47(1.15-3.79) } & Tests of heterogeneity: $l^{2}=46.2 \%$ & $P=0.156$ \\
\hline \multicolumn{4}{|l|}{ CD3: } \\
\hline Study & Hazard ratio & $95 \%$ confidence interval & Weight (\%) \\
\hline Zingg U, 2010 & 0.53 & $0.33-0.84$ & 55.54 \\
\hline Rauser S, 2010 & 0.49 & $0.28-0.85$ & 44.46 \\
\hline \multicolumn{2}{|c|}{ Overall: HR $0.51(0.32-0.70)$} & Tests of heterogeneity: $I^{2}=0.0 \%$ & $P=0.838$ \\
\hline \multicolumn{4}{|l|}{ CD8: } \\
\hline Study & Hazard ratio & $95 \%$ confidence interval & Weight (\%) \\
\hline Zingg U, 2010 & 0.44 & $0.27-0.69$ & 54.36 \\
\hline Dutta S, 2012 & 0.69 & $0.48-0.99$ & 45.64 \\
\hline \multicolumn{2}{|c|}{ Overall: HR $0.55(0.31-0.80)$} & Tests of heterogeneity: $I^{2}=54.5 \%$ & $P=0.138$ \\
\hline \multicolumn{4}{|l|}{ EGFR: } \\
\hline Study & Hazard ratio & $95 \%$ confidence interval & Weight (\%) \\
\hline Ong CA, 2013 & 1.52 & $1.03-2.26$ & 67.93 \\
\hline Wang K, 2007 & 1.93 & $1.24-3.03$ & 32.07 \\
\hline \multicolumn{2}{|c|}{ Overall: HR 1.65 (1.14-2.16) } & Tests of heterogeneity: $I^{2}=0.0 \%$ & $P=0.459$ \\
\hline \multicolumn{4}{|l|}{ p53: } \\
\hline Study & Hazard ratio & $95 \%$ confidence interval & Weight (\%) \\
\hline Moskaluk CA, 1996 & 1.46 & $0.87-2.46$ & 39.61 \\
\hline Cavazzola LT, 2009 & 1.43 & $0.43-4.72$ & 5.44 \\
\hline Madani K, 2010 & 1.64 & $1.10-2.45$ & 54.95 \\
\hline
\end{tabular}

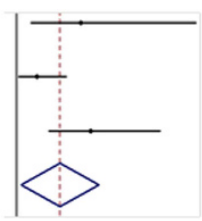

CD3

Rauser S, 2010

Tests of heterogeneity: $I^{2}=0.0 \%$

$P=0.938$
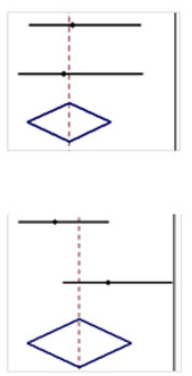

Overall:HR $1.56(1.06-2.06)$

(n)

\begin{tabular}{lccc} 
HER2: & & & \\
Study & Hazard ratio & $95 \%$ confidence interval & Weight (\%) \\
Yoon HH, 2012 & 0.76 & $0.59-0.96$ & 82.38 \\
Phillips BE, 2013 & 0.84 & $0.53-1.33$ & 17.62 \\
Overall: HR 0.77 $(0.61-0.94)$ & Tests of heterogeneity: $I^{2}=0.0 \%$ & $P=0.722$ \\
\hline
\end{tabular}

Overall: HR 0.77 (0.61-0.94

Tests of heterogeneity: $I^{2}=0.0 \%$

\begin{tabular}{lccc} 
LgR5: & & & \\
Study & Hazard ratio & $95 \%$ confidence interval & Weight (\%) \\
Von Rahden BH, 2011 & 2.42 & $1.17-4.99$ & 74.50 \\
Becker L, 2010 & 2.86 & $1.08-7.61$ & 25.50 \\
Overall: HR 2.53 (0.88-4.18) & Tests of heterogeneity: $I^{2}=0.0 \%$ & $P=0.819$ \\
\hline
\end{tabular}

\begin{tabular}{lccc} 
Ki67: & & & \\
Study & Hazard ratio & $95 \%$ confidence interval & Weight (\%) \\
Falkenback D, 2008 & 3.90 & $1.70-9.10$ & 9.84 \\
Dutta S, 2012 & 1.46 & $1.01-2.12$ & 85.21 \\
Evangelou K,2007 & 3.76 & $0.99-11.68$ & 4.96 \\
& & & \\
Overall: HR 1.81 (0.59-3.03) & Tests of heterogeneity: $I^{2}=13.2 \%$ & $P=0.316$ \\
\hline VEGF: & Hazard ratio & $95 \%$ confidence interval & Weight (\%) \\
Study & 0.37 & $0.09-1.44$ & 37.24 \\
Cavazzola LT, 2009 & 0.88 & $0.54-1.42$ & 62.76 \\
Prins MJ, 2012 & & & \\
& & & \\
Overall: HR 0.69 $(0.21-1.17)$ & Tests of heterogeneity: $I^{2}=35.9 \%$ & $P=0.212$ \\
\hline
\end{tabular}
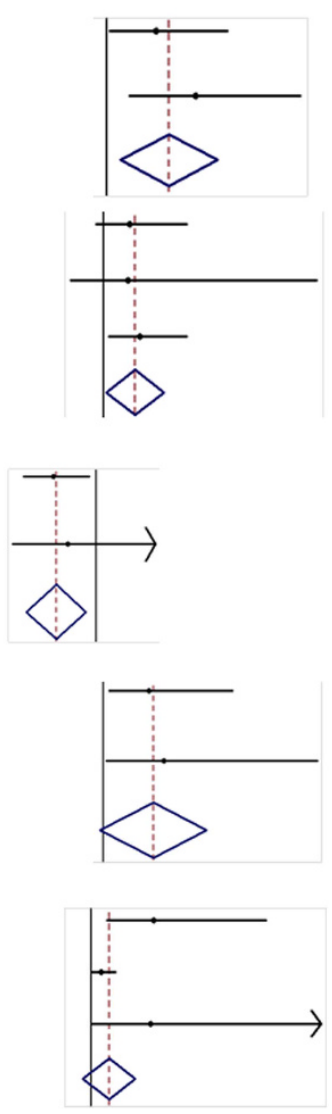

Figure 4. Forest plots with associated hazard ratio (HR) and $95 \%$ confidence interval. Weights calculated using a random effects model. $\mathrm{HR}>1$ implies worse survival with overexpression, $\mathrm{HR}<1$; improved survival (vertical black line indicates HR of 1 ; red vertical dotted line indicates overall HR). A full colour version of this figure is available at the British Journal of Cancer journal online.

receptor and its ligand (McDermott and Atkins, 2013). Two studies were pooled examining CD8 (cytotoxic, T-cell effector), comprising a total of 203 cases (Zingg et al, 2010; Dutta et al, 2012). Moderate heterogeneity is observed $\left(I^{2}=54.5 \%\right)$. Methodological differences may be the cause of the heterogeneity, with observation of increasing $\mathrm{CD} 8+$ count across three tertiles, vs CD8 with a cutoff set for high vs low expression (Zingg et al, 2010; Dutta et al, 2012).

EGFR. EGFR is a receptor tyrosine kinase, shown to have effects on cancer differentiation, proliferation, invasion and metastasis (Grandis and Sok, 2004). EGFR targeting is used in the treatment 
of colorectal cancer and non-small cell lung cancer (Mahipal et al, 2014). A total of 642 patients were pooled from two studies (Wang et al, 2007; Ong et al, 2013). Combined, an overall, slight-negative, prognostic effect of EGFR overexpression was found. This is in agreement with other studies in OAC that were unsuitable for meta-analysis (Mukaida et al, 1991; Yacoub et al, 1997; Lennerz et al, 2011). This effect on prognosis with overexpressed EGFR has been noted in both colorectal cancer and gastric adenocarcinoma (Rego et al, 2010; Hong et al, 2013).

p53. p53 acts as a hub for multiple intra-cellular surveillance systems, constantly reporting on cellular integrity. When stress is detected, a damaged cell can initiate DNA repair, senescence and/ or apoptosis. TP53 mutation can increase protein stability, meaning IHC detection correlates with mutation (Bellini et al, 2012). However IHC does not account for all mutations, with between $52 \%$ and $80 \%$ agreement between IHC and PCR with truncating and missense mutations (Bian et al, 2001). TP53 is the most commonly mutated gene in $\mathrm{OAC}$ and has recently been found to have a mutational frequency that would distinguish between disease stages and thus identify progression towards malignancy (Weaver et al, 2014).

Eleven studies examining p53 were reviewed in full (Flejou et al, 1994; Casson et al, 1995; Sauter et al, 1995; Moskaluk et al, 1996; Hardwick et al, 1997; Casson et al, 1998, 2003; Jiao et al, 2003; Heeren et al, 2004; Cavazzola et al, 2009; Madani et al, 2010). Only three of these were suitable for inclusion in the systematic review and subsequent meta-analysis, containing a total of 268 patients and showing a pooled effect of worse prognosis with increased expression (Moskaluk et al, 1996; Cavazzola et al, 2009; Madani et al, 2010). Good agreement is seen between the three included studies. However, five other studies not included in the review failed to reach significance, suggesting that the prognostic value of p53 may not be as obvious as the meta-analysed results suggest (Duhaylongsod et al, 1995; Coggi et al, 1997; Hardwick et al, 1997; Langer et al, 2006; Falkenback et al, 2008).

HER2. HER2 exhibits extensive homology with EGFR, frequently dimerising with it or another member of the EGFR family HER3 (Wolf-Yadlin et al, 2006). HER2 is overexpressed in a number of cancers and undergoes a ligand-independent activation, with consequent downstream signals involved in proliferation and migration (Wolf-Yadlin et al, 2006). HER2 provides a target for the monoclonal antibody trastuzumab, which has proven efficacy in breast and gastric cancer treatment (Hynes and Lane, 2005; Bang et al, 2010). HER2 targeting in OAC is being assessed in the feasibility arm of the MRC STO3 clinical trial (Okines et al, 2013).

Two studies were suitable for pooling, showing an OS benefit with overexpression of HER2 (Nakamura et al, 1994; Yoon et al, 2012; Phillips et al, 2013). Both studies used the ToGA trial protocol to assess HER2 overexpression, including FISH analysis of ErbB2 gene amplification. This effect has failed to be reproduced by smaller studies, potentially as a result of under powering (Polkowski et al, 1999; Reichelt et al, 2007; Hu et al, 2011; Thompson et al, 2011). The overall protective effect seen here is in contrast to studies investigating OAC using techniques other than IHC, where a negative prognostic effect is noted, as well as in breast cancer, where a dramatically worse prognosis is seen with overexpression (Andrulis et al, 1998; Chan et al, 2012).

LgR5. The R-spondin receptor Lgr5 is a stem cell marker in multiple organs in mice and humans. Single Lgr5 stem cells derived from the intestine can be cultured to build epithelial structures that retain hallmarks of the in vivo epithelium (Sato and Clevers, 2013). In tumours, Lgr5 expression is believed to define cancer stem cells and may have prognostic effects by promoting invasion and metastasis as well as initiating self-renewal pathways (Reya and Clevers, 2005). Despite the vast majority of cancer deaths being attributable to invasion and metastasis, Lgr5 was the only suitable biomarker for meta-analysis with its main function associated with this hallmark of cancer (Becker et al, 2010; von Rahden et al, 2011).

Lgr5 failed to reach statistical significance as a prognostic marker. This was due to the wide, asymmetric CIs, resulting from under powering with only 84 cases in total across the two studies (Becker et al, 2010; von Rahden et al, 2011).

VEGF. VEGF is upregulated in response to hypoxia, acting as a key mediator of angiogenesis and affecting vessel permeability, potentially enhancing haematogenous dissemination (Hicklin and Ellis, 2005). Two studies contributed to VEGF meta-analysis, with a total of 181 patients, producing a non-significant effect on survival (Cavazzola et al, 2009; Prins et al, 2012). Again, few studies have examined prognosis and angiogenesis, with contradictory results seen in small cohorts (Couvelard et al, 2000; Saad et al, 2005). With emerging targeted therapies, further work will be required to confirm whether VEGF is a true driver of cancer aggressiveness (Shah et al, 2011).

Ki67. Despite the common use of Ki67 to index cellular proliferation, its biological function in the tumour remains elusive. It seems to co-localise with ribosomal RNA during mitosis suggesting a role in protein synthesis and, more recently, chromatin remodelling (Bullwinkel et al, 2006).

Here, three studies were pooled, comprising a total of 192 patients (Evangelou et al, 2008; Falkenback et al, 2008; Dutta et al, 2012). A non-significant result was observed. Again, this could be due to a combination of asymmetrical wide CIs in two studies, combined with marginal prognostic value in the other. In breast cancer, increased cellular proliferation index has been studied as a negative prognostic marker and in directing use of chemotherapy against rapidly dividing tumours (Martin et al, 2004; de Azambuja et al, 2007; Yerushalmi et al, 2010). However, Ki67 expression is understudied in $\mathrm{OAC}$, and prognostic significance remains inconclusive.

Publication bias. Within the 214 relevant articles, 92 of these provided HRs and statistical significance, 52 (57\%) of these provided non-significant results. This is in contrast to the final 36 articles that met REMARK inclusion criteria, where only six (18\%) centred on non-significant results. Asymmetry was noted when all data was viewed on a funnel plot (Figure 2) suggesting positive publication bias.

\section{DISCUSSION}

Previous meta-analysis of oesophageal cancer examining individual molecules of prognosis have combined OAC and SCC in addition to using different investigational techniques for analysis (Vallbohmer and Lenz, 2006; Ong et al, 2010; Chan et al, 2012; Chen et al, 2012, 2013; Peng et al, 2013; Gowryshankar et al, 2014). There is consensus that OAC and SCC should be considered as separate biological entities and current clinical trials in oesophageal cancer reflect this approach. To date, this is the first meta-analysis that has synthesised the literature associated with all IHC markers solely in resected OAC. Using a validated prognostic markerreporting tool to inform our strict inclusion and exclusion criteria, we identified 36 high-quality articles providing reliable HRs and CIs (McShane et al, 2005). From these articles, nine markers were suitable for meta-analysis and of these six markers showed significant correlation with survival. These markers were COX-2, CD3, CD8, HER2, EGFR and p53. Several other molecules have been assessed in good quality studies that met the REMARK inclusion criteria, but do not have a second study available for pooling. Of particular interest, MET, B7-H1, CAIX, ANXA1 and 
VEGF-C all showed significant, highly prognostic effects in cohorts containing over 100 cases but still require validation and/or elucidation of the underlying biology.

A number of the molecules identified in this review are related to emerging therapies. Four of the nine meta-analysed markers (COX-2 - celecoxib, EGFR - gefitinib, HER2 trastuzumab and VEGF - bevacizumab) focussed on molecules with targeted therapeutics either already in use or in development, and two lymphocyte markers representing the presence of effectors of anti-tumour immunity, which can be induced by new therapies (Zhang et al, 2003; Galon et al, 2006; Ekman et al, 2008; Mei et al, 2014; Ward et al, 2014). As well as new therapies, there is an increasing interest in the role the cancer microenvironment has in OAC progression (Courrech Staal et al, 2011). Here, CD 3 and CD 8 demonstrate the greatest protective prognostic impact, illustrating the importance of the immune response to OAC. However, IHC analysis of other components of the microenvironment have been largely neglected, for example, only two papers comment on the impact of cancerassociated fibroblasts on prognosis (Laerum et al, 2012; Schoppmann et al, 2013a).

The most striking observation of this meta-analysis is the scarcity of high-quality articles, with 66\% (69 out of 104) of potentially suitable studies not conforming to REMARK criteria. In similar meta-analyses published on the two cancers with worse prognoses than OAC, 83 suitable articles were pooled for pancreatic cancer prognosis, and in lung cancer, enough data were found to analyse 17 markers studied in four or more papers (Zhu et al, 2006; Jamieson et al, 2011; Peng et al, 2013). This suggests that prognostic marker research in OAC is lacking. In addition, despite the majority of patients now receiving some form of neoadjuvant therapy before resection for OAC (Noble et al, 2013), we found this to be poorly reported in these studies. It was therefore impossible to make any attempt to discriminate between markers prognostic after primary resection or after neoadjuvant therapy. Future reports should include a detailed description of the types of multimodal treatment given to patients and preferably include an analysis based on these treatment types.

A trend was noticed towards more robust methodology when authors used larger data sets. The largest study identified used independent generation and validation data sets to confirm the prognostic significance of the novel markers SIRT2 and TRIMM44. The analysis was performed in two different patient cohorts from separate centres and was of high quality (Ong et al, 2013). However, we were unable to include this study in the meta-analysis because the cutoffs used to assess the HRs in the two cohorts were different. SIRT2 and TRIMM44 require validation using the same methodology and cutoffs in another cohort. Despite this, the study by Ong et al (2013) describes a sophisticated approach to the development of a biomarker based on genetic analysis carried through to the protein level. Genome sequencing studies such as the UK ICGC project in OAC (Weaver et al, 2014) will deliver more potential markers of prognosis in selected sub-groups and methods such as those described by Ong et al (2013) will be required to translate these findings into meaningful clinical outcomes.

Authors who appeared more than once in the 214 initial articles, often adhered to REMARK criteria, and provided log-rank or cox regression hazard calculations. This suggests a gradual uptake of REMARK criteria, since it's inception in 2005. Another potential reason for this poorer reporting in smaller studies may be due to more frequent negative results due to inadequate powering. With an overall reluctance towards negative reporting, it is quite possible that these results are left out as redundant data, with the larger data sets having more positive results, and a greater likelihood of publication.(Kyzas et al, 2007)
Limitations. Meta-analysis is able to enhance power that leads to more robust generalisations within a field. However, there are notorious confounding factors (Altman, 2001).

Here, only one study was prospective in design (Madani et al, 2010). Retrospective analysis allows potential issues in reporting and selection bias. With differences in multivariate or univariate analysis, CSS or OS, size of cohorts, cutoffs, primary antibody at different dilutions and occasionally radically different numbers of positive staining cases lead to less validity when combining results. Future work will require multi-centre efforts to gather large enough, prospective cohorts to provide robust clarification of truly prognostic markers.

With this meta-analysis we have only included IHC detectable markers of survival. Both IHC and RT-PCR have their own limitations; however, IHC is seen to be the most practical way to assess protein expression in solid cancers, with IHC survival biomarkers well described in other malignancies (Zhu et al, 2006; Jamieson et al, 2011).

In future work, multivariate modelling will give an insight into interaction between different variables in OAC. In this study, univariate analysis was used preferentially, to limit heterogeneity between methods of producing HRs, as a multivariate HR can be altered by use of different prognostic factors or model types in individual models. In fact, it is likely that a combination of markers will be required to give meaningful prognostic information to an individual patient, perhaps covering multiple Hallmarks of Cancer, rather than considering individual biomarkers in isolation. There are existing data from oesophageal cancer biology to support this strategy (Kadri et al, 2010; Peters et al, 2010; Liu et al, 2014).

\section{CONCLUSION}

Current methods have not delivered clinically useful molecular prognostic biomarkers in OAC. We have highlighted the paucity of good-quality robust studies in this field. This may be because little attention has been focused on OAC research compared with other cancers, or perhaps it is an indication of the molecular complexity of the disease that is only just beginning to be appreciated. The development of new and novel biomarkers in OAC will require understanding of this complexity and in this context IHC alone seems inappropriate. A genome to protein approach would be better suited for the development and subsequent validation of biomarkers. Large collaborative projects with standardised methodology will be required to generate clinically useful biomarkers.

\section{ACKNOWLEDGEMENTS}

This work is supported by a UK Medical Research Council Clinician Scientist grant to TJU. FN is supported by Cancer Research UK.

\section{CONFLICT OF INTEREST}

The authors declare no conflict of interest.

\section{REFERENCES}

Altman DG (2001) Systematic reviews of evaluations of prognostic variables. BMJ 323(7306): 224-228.

Andrulis IL, Bull SB, Blackstein ME, Sutherland D, Mak C, Sidlofsky S, Pritzker KP, Hartwick RW, Hanna W, Lickley L, Wilkinson R, Qizilbash A, Ambus U, Lipa M, Weizel H, Katz A, Baida M, Mariz S, 
Stoik G, Dacamara P, Strongitharm D, Geddie W, McCready D (1998) neu/erbB-2 amplification identifies a poor-prognosis group of women with node-negative breast cancer. Toronto Breast Cancer Study Group. J Clin Oncol 16(4): 1340-1349.

Bang YJ, Van Cutsem E, Feyereislova A, Chung HC, Shen L, Sawaki A, Lordick F, Ohtsu A, Omuro Y, Satoh T, Aprile G, Kulikov E, Hill J, Lehle M, Ruschoff J, Kang YK. To GATI (2010) Trastuzumab in combination with chemotherapy versus chemotherapy alone for treatment of HER2-positive advanced gastric or gastro-oesophageal junction cancer (ToGA): a phase 3, open-label, randomised controlled trial. Lancet 376(9742): 687-697.

Becker L, Huang Q, Mashimo H (2010) Lgr5, an intestinal stem cell marker, is abnormally expressed in Barrett's esophagus and esophageal adenocarcinoma. Dis Esophagus 23(2): 168-174.

Bettstetter M, Berezowska S, Keller G, Walch A, Feuchtinger A, SlottaHuspenina J, Feith M, Drecoll E, Hofler H, Langer R (2013) Epidermal growth factor receptor, phosphatidylinositol-3-kinase catalytic subunit/ PTEN, and KRAS/NRAS/BRAF in primary resected esophageal adenocarcinomas: loss of PTEN is associated with worse clinical outcome Hum Pathol 44(5): 829-836.

Bellini MF, Cadamuro AC, Succi M, Proenca MA, Silva AE (2012) Alterations of the TP53 gene in gastric and esophageal carcinogenesis. J Biomed Biotechnol 2012: 891961.

Bhandari P, Bateman AC, Mehta RL, Stacey BS, Johnson P, Cree IA, Di Nicolantonio F, Patel P (2006) Prognostic significance of cyclooxygenase-2 (COX-2) expression in patients with surgically resectable adenocarcinoma of the oesophagus. BMC Cancer 6: 134.

Bian YS, Osterheld MC, Bosman FT, Benhattar J, Fontolliet C (2001) p53 gene mutation and protein accumulation during neoplastic progression in Barrett's esophagus. Mod Pathol 14(5): 397-403.

Birner P, Jesch B, Friedrich J, Riegler M, Zacherl J, Hejna M, Wrba F, Schultheis A, Schoppmann SF (2011) Carbonic anhydrase IX overexpression is associated with diminished prognosis in esophageal cancer and correlates with Her-2 expression. Ann Surg Oncol 18(12): 3330-3337.

Birner P, Jesch B, Schultheis A, Schoppmann SF (2012) RAF-kinase inhibitor protein (RKIP) downregulation in esophageal cancer and its metastases. Clin Exp Metastasis 29(6): 551-559.

Bullwinkel J, Baron-Luhr B, Ludemann A, Wohlenberg C, Gerdes J, Scholzen T (2006) Ki-67 protein is associated with ribosomal RNA transcription in quiescent and proliferating cells. J Cell Physiol 206(3): 624-635.

Buskens CJ, Van Rees BP, Sivula A, Reitsma JB, Haglund C, Bosma PJ, Offerhaus GJ, Van Lanschot JJ, Ristimaki A (2002) Prognostic significance of elevated cyclooxygenase 2 expression in patients with adenocarcinoma of the esophagus. Gastroenterology 122(7): 1800-1807.

Casson AG, Evans SC, Gillis A, Porter GA, Veugelers P, Darnton SJ, Guernsey DL, Hainaut P (2003) Clinical implications of p53 tumor suppressor gene mutation and protein expression in esophageal adenocarcinomas: results of a ten-year prospective study. J Thorac Cardiovasc Surg 125(5): $1121-1131$.

Casson AG, Kerkvliet N, O’Malley F (1995) Prognostic value of p53 protein in esophageal adenocarcinoma. J Surg Oncol 60(1): 5-11.

Casson AG, Tammemagi M, Eskandarian S, Redston M, McLaughlin J, Ozcelik H (1998) p53 alterations in oesophageal cancer: association with clinicopathological features, risk factors, and survival. Mol Pathol 51(2): 71-79.

Cavazzola LT, Rosa AR, Schirmer CC, Gurski RR, Telles JP, Mielke F, Meurer L, Edelweiss MI, Kruel CD (2009) Immunohistochemical evaluation for P53 and VEGF (Vascular Endothelial Growth Factor) is not prognostic for long term survival in end stage esophageal adenocarcinoma. Rev Col Bras Cir 36(1): 24-34.

Chan DS, Twine CP, Lewis WG (2012) Systematic review and meta-analysis of the influence of HER2 expression and amplification in operable oesophageal cancer. J Gastrointest Surg 16(10): 1821-1829.

Chandra RK, Bentz BG, Haines 3rd GK, Robinson AM, Radosevich JA (2002) Expression of glutathione s-transferase pi in benign mucosa, Barrett's metaplasia, and adenocarcinoma of the esophagus. Head Neck 24(6): 575-581.

Chen M, Cai E, Huang J, Yu P, Li K (2012) Prognostic value of vascular endothelial growth factor expression in patients with esophageal cancer: a systematic review and meta-analysis. Cancer Epidemiol Biomarkers Prev 21(7): 1126-1134.
Chen M, Huang J, Zhu Z, Zhang J, Li K (2013) Systematic review and meta-analysis of tumor biomarkers in predicting prognosis in esophageal cancer. BMC Cancer 13(1): 539.

Coggi G, Bosari S, Roncalli M, Graziani D, Bossi P, Viale G, Buffa R, Ferrero S, Piazza M, Blandamura S, Segalin A, Bonavina L, Peracchia A (1997) p53 protein accumulation and p53 gene mutation in esophageal carcinoma. A molecular and immunohistochemical study with clinicopathologic correlations. Cancer 79(3): 425-432.

Corley DA, Kerlikowske K, Verma R, Buffler P (2003) Protective association of aspirin/NSAIDs and esophageal cancer: a systematic review and metaanalysis. Gastroenterology 124(1): 47-56.

Courrech Staal EF, Smit VT, van Velthuysen ML, Spitzer-Naaykens JM, Wouters MW, Mesker WE, Tollenaar RA, van Sandick JW (2011) Reproducibility and validation of tumour stroma ratio scoring on oesophageal adenocarcinoma biopsies. Eur J Cancer 47(3): 375-382.

Couvelard A, Paraf F, Gratio V, Scoazec JY, Henin D, Degott C, Flejou JF (2000) Angiogenesis in the neoplastic sequence of Barrett's oesophagus. Correlation with VEGF expression. J Pathol 192(1): 14-18.

Dahlin AM, Henriksson ML, Van Guelpen B, Stenling R, Oberg A, Rutegard J, Palmqvist R (2011) Colorectal cancer prognosis depends on T-cell infiltration and molecular characteristics of the tumor. Mod Pathol 24(5): 671-682.

de Azambuja E, Cardoso F, de Castro Jr G, Colozza M, Mano MS, Durbecq V, Sotiriou C, Larsimont D, Piccart-Gebhart MJ, Paesmans M (2007) Ki-67 as prognostic marker in early breast cancer: a meta-analysis of published studies involving 12,155 patients. Br J Cancer 96(10): 1504-1513.

DiMaio MA, Kwok S, Montgomery KD, Lowe AW, Pai RK (2012) Immunohistochemical panel for distinguishing esophageal adenocarcinoma from squamous cell carcinoma: a combination of $\mathrm{p} 63$, cytokeratin 5/6, MUC5AC, and anterior gradient homolog 2 allows optimal subtyping. Hum Pathol 43(11): 1799-1807.

Duhaylongsod FG, Gottfried MR, Iglehart JD, Vaughn AL, Wolfe WG (1995) The significance of c-erb B-2 and p53 immunoreactivity in patients with adenocarcinoma of the esophagus. Ann Surg 221(6): 677-683, discussion 683-684.

Dulak AM, Stojanov P, Peng S, Lawrence MS, Fox C, Stewart C, Bandla S, Imamura Y, Schumacher SE, Shefler E, McKenna A, Carter SL, Cibulskis K, Sivachenko A, Saksena G, Voet D, Ramos AH, Auclair D, Thompson K, Sougnez C, Onofrio RC, Guiducci C, Beroukhim R, Zhou Z, Lin L, Lin J, Reddy R, Chang A, Landrenau R, Pennathur A, Ogino S, Luketich JD, Golub TR, Gabriel SB, Lander ES, Beer DG, Godfrey TE, Getz G, Bass AJ (2013) Exome and whole-genome sequencing of esophageal adenocarcinoma identifies recurrent driver events and mutational complexity. Nat Genet 45(5): 478-486.

Dutta S, Going JJ, Crumley AB, Mohammed Z, Orange C, Edwards J, Fullarton GM, Horgan PG, McMillan DC (2012) The relationship between tumour necrosis, tumour proliferation, local and systemic inflammation, microvessel density and survival in patients undergoing potentially curative resection of oesophageal adenocarcinoma. Br J Cancer 106(4): $702-710$.

Ekman S, Dreilich M, Lennartsson J, Wallner B, Brattstrom D, Sundbom M, Bergqvist M (2008) Esophageal cancer: current and emerging therapy modalities. Expert Rev Anticancer Ther 8(9): 1433-1448.

Evangelou K, Kotsinas A, Mariolis-Sapsakos T, Giannopoulos A, Tsantoulis PK, Constantinides C, Troupis TG, Salmas M, Kyroudis A, Kittas C, Gorgoulis VG (2008) E2F-1 overexpression correlates with decreased proliferation and better prognosis in adenocarcinomas of Barrett oesophagus. J Clin Pathol 61(5): 601-605.

Falkenback D, Nilbert M, Oberg S, Johansson J (2008) Prognostic value of cell adhesion in esophageal adenocarcinomas. Dis Esophagus 21(2): $97-102$.

Fareed KR, Kaye P, Soomro IN, Ilyas M, Martin S, Parsons SL, Madhusudan S (2009) Biomarkers of response to therapy in oesophago-gastric cancer. Gut 58(1): 127-143.

Flejou JF, Paraf F, Potet F, Muzeau F, Fekete F, Henin D (1994) p53 protein expression in Barrett's adenocarcinoma: a frequent event with no prognostic significance. Histopathology 24(5): 487-489.

France M, Drew PA, Dodd T, Watson DI (2004) Cyclo-oxygenase-2 expression in esophageal adenocarcinoma as a determinant of clinical outcome following esophagectomy. Dis Esophagus 17(2): 136-140.

Galon J, Costes A, Sanchez-Cabo F, Kirilovsky A, Mlecnik B, Lagorce-Pages C, Tosolini M, Camus M, Berger A, Wind P, Zinzindohoue F, Bruneval P, Cugnenc PH, Trajanoski Z, Fridman WH, Pages F (2006) Type, density, 
and location of immune cells within human colorectal tumors predict clinical outcome. Science 313(5795): 1960-1964.

Gowryshankar A, Nagaraja V, Eslick GD (2014) HER2 status in Barrett's esophagus \& esophageal cancer: a meta analysis. J Gastrointest Oncol 5(1): $25-35$.

Grandis JR, Sok JC (2004) Signaling through the epidermal growth factor receptor during the development of malignancy. Pharmacol Ther 102(1): $37-46$.

Grimm M, Lazariotou M, Kircher S, Stuermer L, Reiber C, Hofelmayr A, Gattenlohner S, Otto C, Germer CT, von Rahden BH (2010) MMP-1 is a (pre-)invasive factor in Barrett-associated esophageal adenocarcinomas and is associated with positive lymph node status. J Transl Med 8: 99.

Hanahan D, Weinberg RA (2011) Hallmarks of cancer: the next generation. Cell 144(5): 646-674.

Hardwick RH, Barham CP, Ozua P, Newcomb PV, Savage P, Powell R, Rahamin J, Alderson D (1997) Immunohistochemical detection of p53 and c-erbB-2 in oesophageal carcinoma; no correlation with prognosis. Eur J Surg Oncol 23(1): 30-35.

Heeren P, Plukker J, van Dullemen H, Nap R, Hollema H (2005) Prognostic role of cyclooxygenase-2 expression in esophageal carcinoma. Cancer Lett 225(2): 283-289.

Hector A, Montgomery EA, Karikari C, Canto M, Dunbar KB, Wang JS, Feldmann G, Hong SM, Haffner MC, Meeker AK, Holland SJ, Yu J, Heckrodt TJ, Zhang J, Ding P, Goff D, Singh R, Roa JC, Marimuthu A, Riggins GJ, Eshleman JR, Nelkin BD, Pandey A, Maitra A (2010) The Axl receptor tyrosine kinase is an adverse prognostic factor and a therapeutic target in esophageal adenocarcinoma. Cancer Biol Ther 10(10): 1009-1018.

Heeren PA, Kloppenberg FW, Hollema H, Mulder NH, Nap RE, Plukker JT (2004) Predictive effect of p53 and p21 alteration on chemotherapy response and survival in locally advanced adenocarcinoma of the esophagus. Anticancer Res 24(4): 2579-2583.

Hicklin DJ, Ellis LM (2005) Role of the vascular endothelial growth factor pathway in tumor growth and angiogenesis. J Clin Oncol 23(5): 1011-1027.

Higgins JP, Thompson SG, Deeks JJ, Altman DG (2003) Measuring inconsistency in meta-analyses. Br Med J 327(7414): 557-560.

Hong L, Han Y, Yang J, Zhang H, Jin Y, Brain L, Li M, Zhao Q (2013) Prognostic value of epidermal growth factor receptor in patients with gastric cancer: a meta-analysis. Gene 529(1): 69-72.

Hu Y, Bandla S, Godfrey TE, Tan D, Luketich JD, Pennathur A, Qiu X, Hicks DG, Peters JH, Zhou Z (2011) HER2 amplification, overexpression and score criteria in esophageal adenocarcinoma. Mod Pathol 24(7): 899-907.

Hynes NE, Lane HA (2005) ERBB receptors and cancer: the complexity of targeted inhibitors. Nat Rev Cancer 5(5): 341-354.

Jamieson NB, Carter CR, McKay CJ, Oien KA (2011) Tissue biomarkers for prognosis in pancreatic ductal adenocarcinoma: a systematic review and meta-analysis. Clin Cancer Res 17(10): 3316-3331.

Jiao X, Eslami A, Ioffe O, Kwong KF, Henry M, Zeng Q, Refaely Y, Burrows W, Gamliel Z, Krasna MJ (2003) Immunohistochemistry analysis of micrometastasis in pretreatment lymph nodes from patients with esophageal cancer. Ann Thorac Surg 76(4): 996-999, discussion 999-1000.

Kadri SR, Lao-Sirieix P, O’Donovan M, Debiram I, Das M, Blazeby JM, Emery J, Boussioutas A, Morris H, Walter FM, Pharoah P, Hardwick RH, Fitzgerald RC (2010) Acceptability and accuracy of a non-endoscopic screening test for Barrett's oesophagus in primary care: cohort study. BMJ 341: c4372.

Kulke MH, Odze RD, Mueller JD, Wang H, Redston M, Bertagnolli MM (2004) Prognostic significance of vascular endothelial growth factor and cyclooxygenase 2 expression in patients receiving preoperative chemoradiation for esophageal cancer. J Thorac Cardiovasc Surg 127(6): 1579-1586.

Kyzas PA, Denaxa-Kyza D, Ioannidis JP (2007) Almost all articles on cancer prognostic markers report statistically significant results. Eur J Cancer 43(17): 2559-2579.

Laerum OD, Ovrebo K, Skarstein A, Christensen IJ, Alpizar-Alpizar W, Helgeland L, Dano K, Nielsen BS, Illemann M (2012) Prognosis in adenocarcinomas of lower oesophagus, gastro-oesophageal junction and cardia evaluated by uPAR-immunohistochemistry. Int J Cancer 131(3): 558-569.

Lagorce C, Paraf F, Vidaud D, Couvelard A, Wendum D, Martin A, Flejou JF (2003) Cyclooxygenase-2 is expressed frequently and early in Barrett's oesophagus and associated adenocarcinoma. Histopathology 42(5): 457-465.
Langer R, Von Rahden BH, Nahrig J, Von Weyhern C, Reiter R, Feith M, Stein HJ, Siewert JR, Hofler H, Sarbia M (2006) Prognostic significance of expression patterns of c-erbB-2, p53, p16INK4A, p27KIP1, cyclin D1 and epidermal growth factor receptor in oesophageal adenocarcinoma: a tissue microarray study. J Clin Pathol 59(6): 631-634.

Lennerz JK, Kwak EL, Ackerman A, Michael M, Fox SB, Bergethon K, Lauwers GY, Christensen JG, Wilner KD, Haber DA, Salgia R, Bang YJ, Clark JW, Solomon BJ, Iafrate AJ (2011) MET amplification identifies a small and aggressive subgroup of esophagogastric adenocarcinoma with evidence of responsiveness to crizotinib. J Clin Oncol 29(36): 4803-4810.

Liu X, Wong A, Kadri SR, Corovic A, O’Donovan M, Lao-Sirieix P, Lovat LB, Burnham RW, Fitzgerald RC (2014) Gastro-esophageal reflux disease symptoms and demographic factors as a pre-screening tool for Barrett's esophagus. PLoS One 9(4): e94163.

Loos M, Langer R, Schuster T, Gertler R, Walch A, Rauser S, Friess H, Feith M (2011) Clinical significance of the costimulatory molecule B7-H1 in Barrett carcinoma. Ann Thorac Surg 91(4): 1025-1031.

Madani K, Zhao R, Lim HJ, Casson AG (2010) Prognostic value of p53 mutations in oesophageal adenocarcinoma: final results of a 15-year prospective study. Eur J Cardiothorac Surg 37(6): 1427-1432.

Mahipal A, Kothari N, Gupta S (2014) Epidermal growth factor receptor inhibitors: coming of age. Cancer Control 21(1): 74-79.

Mandard AM, Dalibard F, Mandard JC, Marnay J, Henry-Amar M, Petiot JF, Roussel A, Jacob JH, Segol P, Samama G, Ollivier JM, Bonvalot S, Gignoux M (1994) Pathologic assessment of tumor regression after preoperative chemoradiotherapy of esophageal carcinoma. Clinicopathologic correlations. Cancer 73(11): 2680-2686.

Martin B, Paesmans M, Mascaux C, Berghmans T, Lothaire P, Meert AP, Lafitte JJ, Sculier JP (2004) Ki-67 expression and patients survival in lung cancer: systematic review of the literature with meta-analysis. Br J Cancer 91(12): 2018-2025.

McDermott DF, Atkins MB (2013) PD-1 as a potential target in cancer therapy. Cancer Med 2(5): 662-673.

McShane LM, Altman DG, Sauerbrei W, Taube SE, Gion M, Clark GM. Statistics Subcommittee of the NCIEWGoCD (2005) REporting recommendations for tumour MARKer prognostic studies (REMARK). Br J Cancer 93(4): 387-391.

Mei Z, Liu Y, Liu C, Cui A, Liang Z, Wang G, Peng H, Cui L, Li C (2014) Tumour-infiltrating inflammation and prognosis in colorectal cancer: systematic review and meta-analysis. Br J Cancer 110(6): 1595-1605.

Mobius C, Stein HJ, Spiess C, Becker I, Feith M, Theisen J, Gais P, Jutting U, Siewert JR (2005) COX2 expression, angiogenesis, proliferation and survival in Barrett's cancer. Eur J Surg Oncol 31(7): 755-759.

Moher D, Liberati A, Tetzlaff J, Altman DG. Group P (2009) Preferred reporting items for systematic reviews and meta-analyses: the PRISMA statement. BMJ 339: b2535.

Moskaluk CA, Heitmiller R, Zahurak M, Schwab D, Sidransky D, Hamilton SR (1996) p53 and p21(WAF1/CIP1/SDI1) gene products in Barrett esophagus and adenocarcinoma of the esophagus and esophagogastric junction. Hum Pathol 27(11): 1211-1220.

Mukaida H, Toi M, Hirai T, Yamashita Y, Toge T (1991) Clinical significance of the expression of epidermal growth factor and its receptor in esophageal cancer. Cancer 68(1): 142-148.

Nakamura T, Nekarda H, Hoelscher AH, Bollschweiler E, Harbeck N, Becker K, Siewert JR, Harbec N (1994) Prognostic value of DNA ploidy and c-erbB-2 oncoprotein overexpression in adenocarcinoma of Barrett's esophagus. Cancer 73(7): 1785-1794[Erratum appears in Cancer 1994;74(8):2396].

National Oesophago-Gastric Cancer audit (2013) Second Annual Report-2013. The NHS Information Centre: London.

Noble F, Nolan L, Bateman AC, Byrne JP, Kelly JJ, Bailey IS, Sharland DM, Rees CN, Iveson TJ, Underwood TJ, Bateman AR (2013) Refining pathological evaluation of neoadjuvant therapy for adenocarcinoma of the esophagus. World J Gastroenterol 19(48): 9282-9293.

O’Neill JR, Stephens NA, Save V, Kamel HM, Phillips HA, Driscoll PJ, Paterson-Brown S (2013) Defining a positive circumferential resection margin in oesophageal cancer and its implications for adjuvant treatment. Br J Surg 100(8): 1055-1063.

Okines AF, Langley RE, Thompson LC, Stenning SP, Stevenson L, Falk S, Seymour M, Coxon F, Middleton GW, Smith D, Evans L, Slater S, Waters J, Ford D, Hall M, Iveson TJ, Petty RD, Plummer C, Allum WH, Blazeby JM, Griffin M, Cunningham D (2013) Bevacizumab with 
peri-operative epirubicin, cisplatin and capecitabine (ECX) in localised gastro-oesophageal adenocarcinoma: a safety report. Ann Oncol 24(3): 702-709.

Ong CA, Lao-Sirieix P, Fitzgerald RC (2010) Biomarkers in Barrett's esophagus and esophageal adenocarcinoma: predictors of progression and prognosis. World J Gastroenterol 16(45): 5669-5681.

Ong CA, Shapiro J, Nason KS, Davison JM, Liu X, Ross-Innes C, O’Donovan M, Dinjens WN, Biermann K, Shannon N, Worster S, Schulz LK, Luketich JD, Wijnhoven BP, Hardwick RH, Fitzgerald RC (2013) Three-gene immunohistochemical panel adds to clinical staging algorithms to predict prognosis for patients with esophageal adenocarcinoma. J Clin Oncol 31(12): 1576-1582.

Owen J, Punt J, Stranford S (2013) Kuby immunology. 7th edn. W.H. Freeman; Palgrave: NY, USA.

Peng J, Shao N, Peng H, Chen LQ (2013) Prognostic significance of vascular endothelial growth factor expression in esophageal carcinoma: a meta-analysis. JBUON 18(2): 398-406.

Peters CJ, Rees JR, Hardwick RH, Hardwick JS, Vowler SL, Ong CA, Zhang C, Save V, O'Donovan M, Rassl D, Alderson D, Caldas C, Fitzgerald RC. Oesophageal Cancer C, Molecular Stratification Study G (2010) A 4-gene signature predicts survival of patients with resected adenocarcinoma of the esophagus, junction, and gastric cardia. Gastroenterology 139(6): 1995-2004.e15.

Phillips BE, Tubbs RR, Rice TW, Rybicki LA, Plesec T, Rodriguez CP, Videtic GM, Saxton JP, Ives DI, Adelstein DJ (2013) Clinicopathologic features and treatment outcomes of patients with human epidermal growth factor receptor 2-positive adenocarcinoma of the esophagus and gastroesophageal junction. Dis Esophagus 26(3): 299-304.

Polkowski W, van Sandick JW, Offerhaus GJ, ten Kate FJ, Mulder J, Obertop H, van Lanschot JJ (1999) Prognostic value of Lauren classification and c-erbB-2 oncogene overexpression in adenocarcinoma of the esophagus and gastroesophageal junction. Ann Surg Oncol 6(3): 290-297.

Prins MJ, Verhage RJ, ten Kate FJ, van Hillegersberg R (2012) Cyclooxygenase isoenzyme-2 and vascular endothelial growth factor are associated with poor prognosis in esophageal adenocarcinoma. J Gastrointest Surg 16(5): 956-966.

Prins MJ, Verhage RJ, Ruurda JP, ten Kate FJ, van Hillegersberg R (2013) Over-expression of phosphorylated mammalian target of rapamycin is associated with poor survival in oesophageal adenocarcinoma: a tissue microarray study. J Clin Pathol 66(3): 224-228.

Rauser S, Langer R, Tschernitz S, Gais P, Jutting U, Feith M, Hofler H, Walch A (2010) High number of CD45RO + tumor infiltrating lymphocytes is an independent prognostic factor in non-metastasized (stage I-IIA) esophageal adenocarcinoma. BMC Cancer 10: 608.

Rego RL, Foster NR, Smyrk TC, Le M, O'Connell MJ, Sargent DJ, Windschitl $\mathrm{H}$, Sinicrope FA (2010) Prognostic effect of activated EGFR expression in human colon carcinomas: comparison with EGFR status. $\mathrm{Br} J$ Cancer 102(1): 165-172.

Reichelt U, Duesedau P, Tsourlakis M, Quaas A, Link BC, Schurr PG, Kaifi JT, Gros SJ, Yekebas EF, Marx A, Simon R, Izbicki JR, Sauter G (2007) Frequent homogeneous HER-2 amplification in primary and metastatic adenocarcinoma of the esophagus. Mod Pathol 20(1): 120-129.

Reya T, Clevers H (2005) Wnt signalling in stem cells and cancer. Nature 434(7035): 843-850.

Rouvelas I, Zeng W, Lindblad M, Viklund P, Ye W, Lagergren J (2005) Survival after surgery for oesophageal cancer: a population-based study. Lancet Oncol 6(11): 864-870.

Saad RS, El-Gohary Y, Memari E, Liu YL, Silverman JF (2005) Endoglin (CD105) and vascular endothelial growth factor as prognostic markers in esophageal adenocarcinoma. Hum Pathol 36 9: 955-961.

Sato T, Clevers H (2013) Growing self-organizing mini-guts from a single intestinal stem cell: mechanism and applications. Science 340(6137): 1190-1194.

Sauter ER, Keller SM, Erner SM (1995) p53 correlates with improved survival in patients with esophageal adenocarcinoma. J Surg Oncol 58(4): 269-273.

Schoppmann SF, Jesch B, Friedrich J, Jomrich G, Maroske F, Birner P (2012) Phosphorylation of signal transducer and activator of transcription 3 (STAT3) correlates with Her-2 status, carbonic anhydrase 9 expression and prognosis in esophageal cancer. Clin Exp Metastasis 29(6): 615-624.

Schoppmann SF, Jesch B, Riegler MF, Maroske F, Schwameis K, Jomrich G, Birner P (2013a) Podoplanin expressing cancer associated fibroblasts are associated with unfavourable prognosis in adenocarcinoma of the esophagus. Clin Exp Metastasis 30(4): 441-446.

Schoppmann SF, Jesch B, Zacherl J, Riegler MF, Friedrich J, Birner P (2013b) Lymphangiogenesis and lymphovascular invasion diminishes prognosis in esophageal cancer. Surgery 153(4): 526-534.

Shah MA, Jhawer M, Ilson DH, Lefkowitz RA, Robinson E, Capanu M, Kelsen DP (2011) Phase II study of modified docetaxel, cisplatin, and fluorouracil with bevacizumab in patients with metastatic gastroesophageal adenocarcinoma. J Clin Oncol 29(7): 868-874.

Sobin LH, Gospodarowicz MK, Wittekind C (2010) International Union against Cancer. TNM classification of malignant tumours. 7th edn. Wiley-Blackwell: Chichester, UK.

Sterne JA, Egger M, Smith GD (2001) Systematic reviews in health care: Investigating and dealing with publication and other biases in metaanalysis. BMJ 323(7304): 101-105.

Streppel MM, Vincent A, Mukherjee R, Campbell NR, Chen SH, Konstantopoulos K, Goggins MG, Van Seuningen I, Maitra A, Montgomery EA (2012) Mucin 16 (cancer antigen 125) expression in human tissues and cell lines and correlation with clinical outcome in adenocarcinomas of the pancreas, esophagus, stomach, and colon. Hum Pathol 43(10): 1755-1763.

Thompson SK, Sullivan TR, Davies R, Ruszkiewicz AR (2011) Her-2/neu gene amplification in esophageal adenocarcinoma and its influence on survival. Ann Surg Oncol 18(7): 2010-2017.

Tuynman JB, Buskens CJ, Kemper K, ten Kate FJ, Offerhaus GJ, Richel DJ, van Lanschot JJ (2005) Neoadjuvant selective COX-2 inhibition downregulates important oncogenic pathways in patients with esophageal adenocarcinoma. Ann Surg 242(6): 840-849, discussion 849-50.

Tuynman JB, Lagarde SM, Ten Kate FJ, Richel DJ, van Lanschot JJ (2008) Met expression is an independent prognostic risk factor in patients with oesophageal adenocarcinoma. Br J Cancer 98(6): 1102-1108.

Vallbohmer D, Lenz HJ (2006) Predictive and prognostic molecular markers in outcome of esophageal cancer. Dis Esophagus 19(6): 425-432.

von Rahden BH, Kircher S, Lazariotou M, Reiber C, Stuermer L, Otto C, Germer CT, Grimm M (2011) LgR5 expression and cancer stem cell hypothesis: clue to define the true origin of esophageal adenocarcinomas with and without Barrett's esophagus? J Exp Clin Cancer Res 30: 23.

Wang KL, Wu TT, Choi IS, Wang H, Resetkova E, Correa AM, Hofstetter WL, Swisher SG, Ajani JA, Rashid A, Albarracin CT (2007) Expression of epidermal growth factor receptor in esophageal and esophagogastric junction adenocarcinomas: association with poor outcome. Cancer 109(4): 658-667.

Wang KL, Wu TT, Resetkova E, Wang H, Correa AM, Hofstetter WL, Swisher SG, Ajani JA, Rashid A, Hamilton SR, Albarracin CT (2006) Expression of annexin A1 in esophageal and esophagogastric junction adenocarcinomas: association with poor outcome. Clin Cancer Res 12(15): 4598-4604.

Ward MJ, Thirdborough SM, Mellows T, Riley C, Harris S, Suchak K, Webb A, Hampton C, Patel NN, Randall CJ, Cox HJ, Jogai S, Primrose J, Piper K, Ottensmeier CH, King EV, Thomas GJ (2014) Tumourinfiltrating lymphocytes predict for outcome in HPV-positive oropharyngeal cancer. Br J Cancer 110(2): 489-500.

Ward S, Scope A, Rafia R, Pandor A, Harnan S, Evans P, Wyld L (2013) Gene expression profiling and expanded immunohistochemistry tests to guide the use of adjuvant chemotherapy in breast cancer management: a systematic review and cost-effectiveness analysis. Health Technol Assess 17(44): 1-302.

Waterman TA, Hagen JA, Peters JH, DeMeester SR, Taylor CR, Demeester TR (2004) The prognostic importance of immunohistochemically detected node metastases in resected esophageal adenocarcinoma. Ann Thorac Surg 78(4): 1161-1169.

Weaver JM, Ross-Innes CS, Shannon N, Lynch AG, Forshew T, Barbera M, Murtaza M, Ong CA, Lao-Sirieix P, Dunning MJ, Smith L, Smith ML, Anderson CL, Carvalho B, O'Donovan M, Underwood TJ, May AP, Grehan N, Hardwick R, Davies J, Oloumi A, Aparicio S, Caldas C, Eldridge MD, Edwards PA, Rosenfeld N, Tavare S, Fitzgerald RC. OCCAMMS Consortium (2014) Ordering of mutations in preinvasive disease stages of esophageal carcinogenesis. Nat Genet 46(8): 837-843.

Wijnhoven BP, Pignatelli M, Dinjens WN, Tilanus HW (2005) Reduced p120ctn expression correlates with poor survival in patients with adenocarcinoma of the gastroesophageal junction. J Surg Oncol 92(2): $116-123$.

Wolf-Yadlin A, Kumar N, Zhang Y, Hautaniemi S, Zaman M, Kim HD, Grantcharova V, Lauffenburger DA, White FM (2006) Effects of HER2 
overexpression on cell signaling networks governing proliferation and migration. Mol Sys Biol 2: 54.

Xie LX, Zhai TT, Yang LP, Yang E, Zhang XH, Chen JY, Zhang H (2013) Lymphangiogenesis and prognostic significance of vascular endothelial growth factor $\mathrm{C}$ in gastro-oesophageal junction adenocarcinoma. Int $J$ Exp Pathol 94(1): 39-46.

Yacoub L, Goldman H, Odze RD (1997) Transforming growth factor-alpha, epidermal growth factor receptor, and MiB-1 expression in Barrett'sassociated neoplasia: correlation with prognosis. Mod Pathol 10(2): $105-112$.

Yendamuri S, Huang M, Malhotra U, Warren GW, Bogner PN, Nwogu CE, Groman A, Demmy TL (2013) Prognostic implications of signet ring cell histology in esophageal adenocarcinoma. Cancer 119(17): 3156-3161.

Yerushalmi R, Woods R, Ravdin PM, Hayes MM, Gelmon KA (2010) Ki67 in breast cancer: prognostic and predictive potential. Lancet Oncol 11(2): 174-183.

Yoon HH, Shi Q, Sukov WR, Wiktor AE, Khan M, Sattler CA, Grothey A, Wu TT, Diasio RB, Jenkins RB, Sinicrope FA (2012) Association of HER2/ErbB2 expression and gene amplification with pathologic features and prognosis in esophageal adenocarcinomas. Clin Cancer Res 18(2): 546-554.

Zhang L, Conejo-Garcia JR, Katsaros D, Gimotty PA, Massobrio M, Regnani G, Makrigiannakis A, Gray H, Schlienger K, Liebman MN, Rubin SC, Coukos G (2003) Intratumoral T cells, recurrence, and survival in epithelial ovarian cancer. $N$ Engl J Med 348(3): 203-213.

Zhu CQ, Shih W, Ling CH, Tsao MS (2006) Immunohistochemical markers of prognosis in non-small cell lung cancer: a review and proposal for a multiphase approach to marker evaluation. J Clin Pathol 59(8): $790-800$.

Zingg U, Montani M, Frey DM, Dirnhofer S, Esterman AJ, Went P, Oertli D (2010) Tumour-infiltrating lymphocytes and survival in patients with adenocarcinoma of the oesophagus. Eur J Surg Oncol 36(7): 670-677.

cc)(1)(2) This work is licensed under the Creative Commons Attribution-Non-Commercial-Share Alike 4.0 International License. To view a copy of this license, visit http:// creativecommons.org/licenses/by-nc-sa/4.0/

Supplementary Information accompanies this paper on British Journal of Cancer website (http://www.nature.com/bjc) 